\title{
Low-cost tissue simulating phantoms with adjustable wavelength-dependent scattering properties in the visible and infrared ranges
}

Rolf B. Saager

Alan Quach

Rebecca A. Rowland

Melissa L. Baldado

Anthony J. Durkin 


\title{
Low-cost tissue simulating phantoms with adjustable wavelength-dependent scattering properties in the visible and infrared ranges
}

\author{
Rolf B. Saager,* Alan Quach, Rebecca A. Rowland, Melissa L. Baldado, and Anthony J. Durkin \\ University of California, Irvine, 1002 Health Sciences Road, Irvine, California 92612, United States
}

\begin{abstract}
We present a method for low-cost fabrication of polydimethylsiloxane (PDMS) tissue simulating phantoms with tunable scattering spectra, spanning visible, and near-infrared regimes. These phantoms use optical polishing agents (aluminum oxide powders) at various grit sizes to approximate in vivo tissue scattering particles across multiple size distributions (range: 17 to $3 \mu \mathrm{m}$ ). This class of tunable scattering phantoms is used to mimic distinct changes in wavelength-dependent scattering properties observed in tissue pathologies such as partial thickness burns. Described by a power-law dependence on wavelength, the scattering magnitude of these phantoms scale linearly with particle concentration over a physiologic range $\left[\mu_{s}^{\prime}=\left(0.5\right.\right.$ to $\left.\left.2.0 \mathrm{~mm}^{-1}\right)\right]$ whereas the scattering spectra, specific to each particle size distribution, correlate to distinct exponential coefficients (range: 0.007 to 0.32 ). Aluminum oxide powders used in this investigation did not detectably contribute to the absorption properties of these phantoms. The optical properties of these phantoms are verified through inverse adding-doubling methods and the tolerances of this fabrication method are discussed. (๑) The Authors. Published by SPIE under a Creative Commons Attribution 3.0 Unported License. Distribution or reproduction of this work in whole or in part requires full attribution of the original publication, including its DOI. [DOI: 10.1117/1.JBO.21.6.067001]
\end{abstract}

Keywords: tissue phantom; optical properties; tissue spectroscopy; layered media.

Paper 160222TNR received Apr. 8, 2016; accepted for publication May 23, 2016; published online Jun. $13,2016$.

\section{Introduction}

Tissue simulating phantoms can provide a valuable intermediary platform for the evaluation of the performance of optical and spectroscopic instruments. These phantoms can be prepared in a deterministic fashion, in which the absorption and scattering properties can be independently determined and/or modulated. This presents an opportunity to evaluate both the performance and sensitivity of optical spectroscopic methods and instrumentation across the full range of anticipated tissue optical properties and structures under controlled experimental conditions. There are several approaches and media that can be used to mimic various aspects of tissue absorption and scattering (e.g., light transport), each with distinct advantages and limitations. ${ }^{1}$

Polydimethylsiloxane (PDMS) is a commonly used medium to fabricate phantoms that approximate the optical properties of tissue. ${ }^{2,3}$ The refractive index of PDMS is 1.43 at $650 \mathrm{~nm}$ which is in close approximation to that of most tissue, which is typically assigned a refractive index of 1.4. ${ }^{4,5}$ PDMS is optically clear across both visible and near-infrared wavelength regimes (with the exceptions of weak absorption peaks at $~ 912$ and $\sim 1020 \mathrm{~nm}$ associated with vibration absorption bands from silicone). ${ }^{6}$ As diffuse reflectance spectroscopy is primarily governed by optical absorption and scattering, this medium presents opportunities for the incorporation of dyes that would modulate only the absorption properties of the medium- and (sub-)micronsized particles of differing refractive index that would modulate only the scattering properties. PDMS is stable and durable,

*Address all correspondence to: Rolf B. Saager, E-mail: rsaager@uci.edu allowing for use over an extended period of time as well as making it simple to transport between study locations. Finally, the deformability of cured PDMS enables fabrication of phantoms that can be used in both contact and noncontact optical techniques. ${ }^{3}$

Unfortunately, PDMS is hydrophobic and tissue chromophores tend to be soluble in water. ${ }^{1}$ This presents significant challenges in embedding this medium with sources of optical contrast that faithfully represent the spectral properties of tissue across visible and near-infrared ranges. Recent efforts, however, have led to the development of methods to provide a closer approximation to realistic tissue simulating phantoms by (a) incorporating absorbing agents that more faithfully mimic in vivo tissue chromophores that span across both the visible and near-infrared regimes ${ }^{7,8}$ and (b) to account for the tissue's multilayered structure, each with distinct optical properties. ${ }^{8}$

Whereas considerable effort has been directed toward identifying and incorporating dyes and pigments to mimic the absorption properties of tissues in PDMS, ${ }^{2,3,5,7-10}$ agents to mimic differential scattering properties remain largely unexplored. It has been shown that the wavelength-dependent scattering spectrum can be described as a simple power-law relationship, where the exponential coefficient can be empirically related to the mean size/distribution of scatterers in tissue. ${ }^{11}$ Typically, titanium oxides $\left(\mathrm{TiO}_{2}\right)$ have been the scattering agent of choice for these PDMS phantoms. ${ }^{2,3,8,9}$ As a common ingredient in cosmetic makeups and lotions, the size and distribution of these $\mathrm{TiO}_{2}$ particles are similar to a large majority of sub-/extracellular structures that scatter light in normal skin. When employed however, these oxide particles can mimic only a single mean particle size and distribution of scattering objects. In this case, wavelength-dependent scattering is 
constrained to a single spectral shape; only the magnitude may be scaled by the concentration of particles within the medium.

Recent investigational studies have indicated that single scatter size distributions in phantoms may not be sufficient to describe bulk tissue structure for an array of tissue pathologies. ${ }^{10,12-17}$ For instance, partial thickness burns exhibit significant cellular damage and collagen denaturation that will significantly alter the wavelength-dependent scattering properties toward large Mie-like scatterer distributions in both visible and near-infrared regimes due to changes in its structure. ${ }^{12}$ Additionally, these structural changes in tissue morphology may be focal and occupy only discrete subvolumes of the total amount of tissue interrogated by optical spectroscopic techniques.

We present a process for developing PDMS-based phantoms with tunable scattering optical properties that can be used in optical system and/or analysis method evaluation and performance testing. A range of inexpensive aluminum oxide powders, each with distinct mean particle sizes and distributions, is used as scattering agents embedded in PDMS to evaluate whether these commercially available low-cost particles are sufficient to mimic wavelength-dependent changes in scattering spectra observed in in vivo tissue pathologies, such as partial thickness burns. Methods for fabrication of these phantoms as thin layers with differentiated scattering properties are also presented as a means to evaluate measurements where the affected scattering changes reside within subvolumes of tissue interrogated by optical methods.

\section{Method and Materials}

\subsection{Materials Used}

For this investigation, we used the two-part P-4 formulation of PDMS (Eager Plastics). The P-4 formulation of PDMS is optically clear and polymerizes (vulcanizes) at room temperature via the addition of a curing agent at a 10:1 ratio of PDMS to curing agent. Once the curing agent is added to the raw PDMS there is an approximate $60 \mathrm{~min}$ window to manipulate the mixture before the PDMS begins to set. In order for the PDMS to fully cure, however, several days are required (up to $\sim 7$ days). The total cure time is dependent on the total volume of the phantom prepared and the relative volume of external dyes and particles embedded in the PDMS.

We have selected white aluminum oxide powders (Panadyne, Inc) as potential scattering agents for this investigation as they are a commercially available material and inexpensive (\$5-10 per pound). Commonly used as optical polishing agents, they are manufactured in a variety of forms (e.g., slurries and powders) and degrees of purity (often described by their appearance, e.g., brown, gray, and white). In the context of this investigation, we consider the powdered form of $\mathrm{Al}_{2} \mathrm{O}_{3}$ in its higher purity, i.e., white, in order to minimize any potential contribution to absorption from these particles. These powders are manufactured to specific grit sizes. The grit values denote a mean particle size and distribution that that is related to the amount (coarseness) of material removal when these powders are used for polishing. Here, the larger the grit, the finer the polishing and the smaller the particles.

In this study, we evaluate the differential scattering properties of a range of $\mathrm{Al}_{3} \mathrm{O}_{2}$ grits suspended in PDMS. Specifically, we have selected grit sizes of 400,800,1000, and 1200, which correspond to manufacturer specified mean particle sizes of 17.3,
Table 1 Amounts of $\mathrm{Al}_{2} \mathrm{O}_{3}$ particles (determined by mass) added to $100 \mathrm{ml}$ PDMS to fabricate grit-size-specific phantoms of differing concentrations (i.e., magnitude of scattering) across a physiologically relevant range in skin.

\begin{tabular}{lccc} 
& \multicolumn{4}{c}{ Concentrations of $\mathrm{Al}_{2} \mathrm{O}_{3}$ per 100 ml PDMS } \\
\cline { 2 - 4 } & "Low" (g) & "Mid" (g) & "High" (g) \\
\hline 400 grit & 7 & 10 & 14 \\
800 grit & 3 & 5 & 9 \\
1000 grit & 2 & 4 & 6 \\
1200 grit & 1.5 & 3 & 5 \\
\hline
\end{tabular}

$6.5,4.5$, and $3.0 \mu \mathrm{m}$, respectively. In addition to characterizing the scattering spectra that result from each grit-size distribution, three concentrations of powders specific to each grit-size distribution will be fabricated in PDMS to verify whether the magnitude of scattering scales linearly with particle concentration (Table 1).

\subsubsection{Fabrication process}

While this fabrication process generally follows our previously published recipe for PDMS phantoms, ${ }^{3}$ there are some adaptations to this process that warrant a full description of this process below.

Multiple grit-sized batches of aluminum oxide phantoms were prepared following the same recipe and mixing protocol; only the quantity (mass) of aluminum oxide varied with each batch and grit size to cover a range of scattering values anticipated in tissue. For this investigation, $100 \mathrm{ml}$ of PDMS and $10 \mathrm{ml}$ curing agent were prepared for each batch of grit/concentration specific phantom material. From each batch, both a thin layer phantom $(\sim 1 \mathrm{~mm}$ thick $)$ and "semi-infinite" phantom (40 mm thick) were molded.

The raw PDMS and curing agent were measured out in a separate disposable beaker by volume $(100$ and $10 \mathrm{ml}$, respectively). The $\mathrm{Al}_{2} \mathrm{O}_{3}$ was measured out by mass, specific to grit and concentration (Table 1). The $\mathrm{Al}_{2} \mathrm{O}_{3}$ was then added directly to the raw PDMS and mixed vigorously using a disposable Vshaped spatula (Bel-Art, F37840) by hand for $\sim 10 \mathrm{~min}$ to evenly distribute the particles throughout the viscous PDMS. (Note that this is a deviation from our previously published PDMS recipes, ${ }^{2,3,8}$ where the scattering agents were sonicated first to ensure that the particles are monodisbursed. Given that these $\mathrm{Al}_{2} \mathrm{O}_{3}$ particles are larger than $\mathrm{TiO} 2$, we have assumed and subsequently verified (see Fig. 1) that they do not aggregate in their powdered form and hence sonication is unnecessary). The curing agent is then added to the PDMS and manually mixed for $\sim 10 \mathrm{~min}$. Since PDMS is a viscous material, manual manipulation and mixing will incorporate air bubbles into the compound. In order to evacuate the air bubbles introduced during the mixing process, the compound was placed in a vacuum chamber (4.7 1 polycarbonate vacuum chamber, Nalgene). After $20 \mathrm{~min}$, the bottom $60 \%$ to $75 \%$ of the PDMS has been cleared of bubbles, which permits the molding of thin layer $(1 \mathrm{~mm})$ phantoms by the process described below. For thick phantoms $(40 \mathrm{~mm})$, the phantom mixture is returned to the vacuum chamber to continue evacuating the remaining air 
(a)

(b)

(c)

(d)

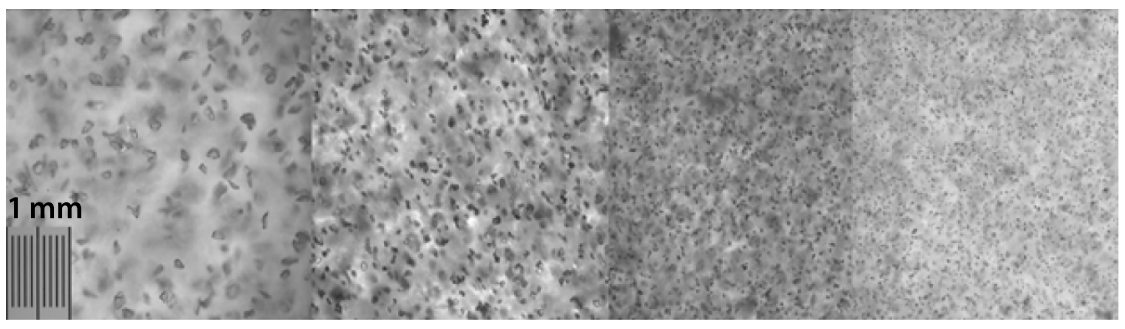

Fig. 1 White light microscopy images of representative phantoms using (a) 400 grit, (b) 800 grit, (c) 1000

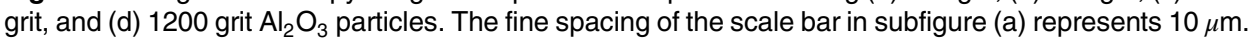

bubbles before the compound begins to fully polymerize. After $24 \mathrm{~h}$, the surface of the phantom will have solidified. A smooth top surface is indicative of the complete removal of all air from the compound.

\subsubsection{Fabrication of individual layer structures}

Once the mixture has been mostly evacuated of air bubbles ( $>\sim 60 \%$ to $75 \%$ ), it is taken out of the vacuum chamber. A 5-ml irrigation syringe (without a needle) is plunged into the mixture, below the region where the remaining air bubbles reside. Using this syringe, a specific volume of the mixture can be extracted and transferred to a plastic petri dish where it is evenly distributed by gravitational forces for samples $>300 \mu \mathrm{m}$ thick (or through the assistance of low-speed spin coating methods, which are not applicable in this particular investigation) and left to cure on a flat, level surface. The precise volume of mixture extracted can be determined by knowing the diameter of the dish and the desired thickness of the cured phantom.

For example, in this investigation, we fabricated thin layer phantoms that are $1 \mathrm{~mm}$ thick, using $60 \mathrm{~mm}$ diameter, generic plastic petri dishes. Since the PDMS does not measurably change in volume during the curing process, we extracted $2.8 \mathrm{ml}$ of the mixture for each thin phantom fabricated here. The percentage error of the thicknesses ranged $<5 \%$ spatially within a single phantom. The variance in mean thickness varied $\sim 10 \%$ between fabrication batches using this simplified approach. These tolerances were determined by digital caliper measurements, sampled at five locations of the thin phantoms.

\subsection{Testing and Evaluation Methods}

In order to characterize and evaluate whether the use of $\mathrm{Al}_{2} \mathrm{O}_{3}$ powders can be utilized as a means to provide distinct/differentiated scattering spectra based on mean particle size distributions, we have employed two optical systems to measure a range of attributes of these fabricated phantoms. These included (1) a bright-field microscopy setup and (2) an integrating sphere-based system. These are described below.

\subsubsection{Visualization of particles in polydimethylsiloxane}

White-light (bright-field) microscopy was used to image just below the surface of each of the thin samples prepared (each grit size at each concentration). We used a white-light microscope (Olympus BH-2 BHTU) at $10 \times$ magnification. From these images, we can qualitatively assess a number of attributes: (1) visualize the shapes and size distributions of particles in each phantom, (2) verify whether or not the particles aggregate within the cured PDMS medium, and (3) verify whether there was any redistribution/settling of the particles during the curing process (by imaging both top and bottom of each phantom).

Additionally, a custom image analysis code was developed to quantitatively estimate the particle size distribution, specific to each grit size. Utilizing the image processing tool box from MATLAB $^{\circledR}$ (Mathworks, Inc.), a simple edge detection scheme was used to identify the boundaries of the individual particles, while rejecting particles that were out of focus. The identified particles where then filled and the areas were calculated for each. Whereas the particles were irregularly shaped, for the simple task of estimating mean particle sizes, we assume that a firstorder approximation of the particles as spheres.

\subsubsection{Determination of diffuse spectral optical properties}

A custom integrating sphere system has been set up at the Beckman Laser Institute to measure the broadband transmittance and reflectance of thin turbid media over a spectral range of 450 to $1000 \mathrm{~nm}$ at $\sim 1 \mathrm{~nm}$ resolution. ${ }^{8,18}$ Placing thin samples at either entrance or exit ports allows this system to measure the total transmittance and reflectance, respectively. Three separate measurements were made per sample, each at a distinct spatial location per sample, in order to provide measures of measurement variance.

Inverse adding-doubling (IAD) is used to calculate the wavelength-dependent optical properties (e.g., absorption and reduced scattering coefficients) from all the thin samples. ${ }^{19}$ The IAD method calculates optical properties (i.e., absorption and scattering coefficients) based on measurements of total reflectance and transmittance via an integrating sphere. Additional physical properties, such as the sample thickness, index of refraction of the medium, $n$, and scattering anisotropy factor, $g$, are also required in order to use the IAD approach to calculate the absorption and scattering coefficients. For this investigation, we have assumed an index of refraction for PDMS to be 1.43 , based on previous literature. ${ }^{3,5}$ We also assumed that the anisotropy factor was between 0.8 and 0.9 . However by preparing samples that are $1 \mathrm{~mm}$ thick, and given the anticipated reduced scattering values of these phantoms, we anticipate that scattered light in both reflectance and transmittance geometries will be primarily isotropic, thereby minimizing any influence from anisotropic scattering signals. This will also be verified in the results section.

From these measurements, we can quantitatively (1) verify whether particle concentration scales linearly the magnitude of the scattering for each grit size, (2) determine the wavelength-dependent scattering properties, specific to each grit size of $\mathrm{Al}_{2} \mathrm{O}_{3}$ particles, and lastly (3) determine what, if any, 
absorption features that these phantoms provide and whether they are concentration dependent.

\section{Results}

\subsection{Microscopy}

The images show the grit-size-specific distributions of $\mathrm{Al}_{2} \mathrm{O}_{3}$ particles in PDMS. From these images, we can qualitatively assess that none of these grit sizes demonstrates any observable aggregation or clumping of particles. Hence the typical sonication step required when using $\mathrm{TiO}_{2}$ powders in PDMS phantom fabrication is not necessary. It is also obvious from these images that the actual shapes of these particles are complex and irregular. Whereas scattering objects in tissue may be modeled by Mie theory, it is worth noting that the jaggedness of these oxide particles may present additional scattering effects (e.g., multiple scattering cross-sections in addition to the mean particle size and distribution). The implications of this will be detailed in the discussion section. Lastly, these images demonstrate that there are distinct particle size distributions for each grit size.

Additionally, from these images, we have run simple imaging processing routines to verify the manufacturer specified mean particle size distributions, specific to each grit size (Table 2). Image processing results from these microscopy images were in general agreement with manufacturer specifications. The mean particle size estimation generally differed by $<1 \mu \mathrm{m}$ between the manufacturer specifications and the image processing results. It was, however, observed that the particle size average was larger for the image processing results than for the manufacturer specifications.

\subsection{Inverse Adding-Doubling (Spectral) Results}

For this investigation, we have chosen to evaluate the fabrication of these variable scatter-size phantoms in the context of diffuse, multiple scattered light. To that end, we have adopted an established model for wavelength-dependent scattering, described by Mourant et.al., ${ }^{11}$ as a means to evaluate the spectral response of these phantoms. This model, indicated in Eq. (1), describes scattering spectra as a simple power-law, where the magnitude of the scattering spectrum can be related to the density of scattering objects within the volume interrogated $(A)$ and the "slope" of the spectrum can be related to the mean size distribution of scattering object $(b)$.

$\mu_{\mathrm{s}}^{\prime}(\lambda)=A * \lambda^{-b}$.

It is worth noting that we have made the following assumptions in the context of this power-law model for scattering: (1) the

Table 2 Comparison of particle diameter and size distribution (std) between manufacturing specifications and microscopy image processing estimations.

\begin{tabular}{lcc}
$\begin{array}{l}\text { Grit-size } \\
\text { (per label) }\end{array}$ & $\begin{array}{c}\text { Manufacturers } \\
\text { specifications }( \pm \text { std })\end{array}$ & $\begin{array}{c}\text { Determined via } \\
\text { microscopy }( \pm \text { std })\end{array}$ \\
\hline 400 grit $(\mu \mathrm{m})$ & $17.3 \pm 8.0$ & $17.1 \pm 11.0$ \\
800 grit $(\mu \mathrm{m})$ & $6.5 \pm 3.0$ & $6.7 \pm 4.9$ \\
1000 grit $(\mu \mathrm{m})$ & $4.5 \pm 1.0$ & $4.1 \pm 2.8$ \\
1200 grit $(\mu \mathrm{m})$ & $3.0 \pm 1.0$ & $3.8 \pm 2.3$ \\
\hline
\end{tabular}

index of refraction is assumed to be constant across all wavelengths and (2) scattering is isotropic (diffuse) for the 1-mm thick phantoms measured by the integrating sphere so the effect of anisotropy $(g)$ is minimal. For the purposes of the IAD model, we assumed a $g$ value of both 0.8 and 0.9 ; however, the reduced scattering coefficient values calculated under those two conditions did not vary beyond measurement noise for the $1 \mathrm{~mm}$ thick samples.

Figure 2 depicts a linear relationship between the concentration of $\mathrm{Al}_{2} \mathrm{O}_{3}$ particles and magnitude of scattering at $650 \mathrm{~nm}$. From this data, it is possible to establish a grit-specific, look-up table that correlates the amount of $\mathrm{Al}_{2} \mathrm{O}_{3}$ to mix with PDMS in order to produce desired reduced scattering coefficients at $650 \mathrm{~nm}$. For the 400 grit powders, we have determined a ratio of $0.112 \mathrm{~g}$ of $\mathrm{Al}_{2} \mathrm{O}_{3}$ per ml PDMS per each $1 \mathrm{~mm}^{-1}$ of reduced scattering. Similarly, 800, 1000, and 1200 grits have linear coefficients of $0.045,0.024,0.016 \mathrm{~g} / \mathrm{ml} / \mathrm{mm}^{-1}$, respectively. From this figure, we can demonstrate that concentration of $\mathrm{Al}_{2} \mathrm{O}_{3}$ particles (scatter density) tracks with the $A$ parameter of the power-law model [Eq. (1)] within a physiologically relevant range, as expected.

Figure 3 shows the reduced scattering coefficient spectra for each grit-size sets of phantoms. The spectra have been normalized to 1 at $450 \mathrm{~nm}$ so all measurements (including phantoms with differing concentrations of $\mathrm{Al}_{2} \mathrm{O}_{3}$ ) may be used and the shape (i.e., "b," scatter slope) may be directly compared, independent of the particle concentration. Fitting these spectra to the power-law model described by Eq. (1), the scatter slope $(b)$ can be determined. Table 3 shows the exponential coefficients determined by the fits. As expected, there was a progression of increasing values for $b$, as the mean particle size diminished. Additionally, these particles are substantially larger than $\mathrm{TiO}_{2}$ particles and typical scattering objects from healthy tissue, which have scatter slopes of $\sim 1.25^{2,3,8}$ and $\sim 1.2 \pm 0.23,{ }^{10,20}$ respectively. The departure of these $\mathrm{Al}_{2} \mathrm{O}_{3}$ phantoms from the scattering properties of healthy tissue corresponds to spectral scattering changes observed within tissue pathologies, such as burns. $^{12}$

Figure 4 shows the respective absorption coefficients $\left(\mu_{\mathrm{a}}\right)$ from all grit sizes. Included in this figure are all three concentrations investigated specific to each grit size without any

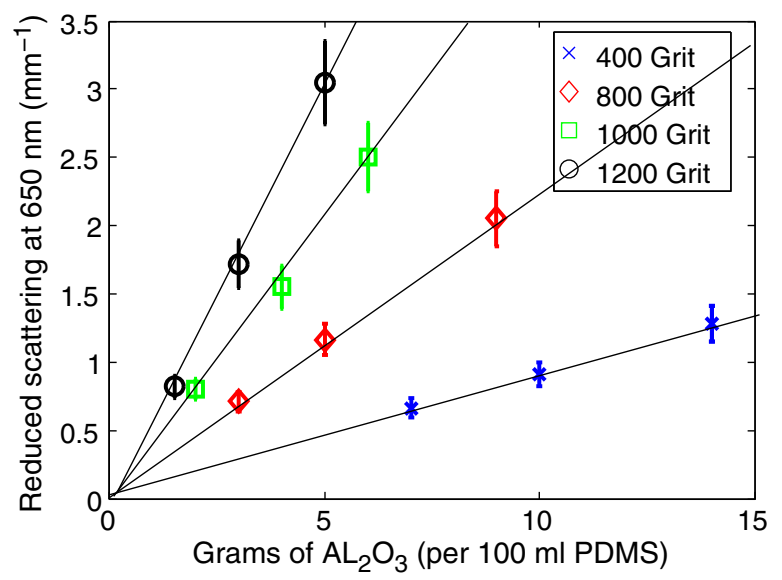

Fig. 2 Concentration of $\mathrm{Al}_{2} \mathrm{O}_{3}$ particles scale linearly with magnitude of scattering over a physiological range. Here each grit-size distribution of $\mathrm{Al}_{2} \mathrm{O}_{3}$ particles was fabricated and measured at three concentrations (based on mass/100 $\mathrm{ml}$ PDMS). The reduced scattering values at $650 \mathrm{~nm}$ are reported here. 


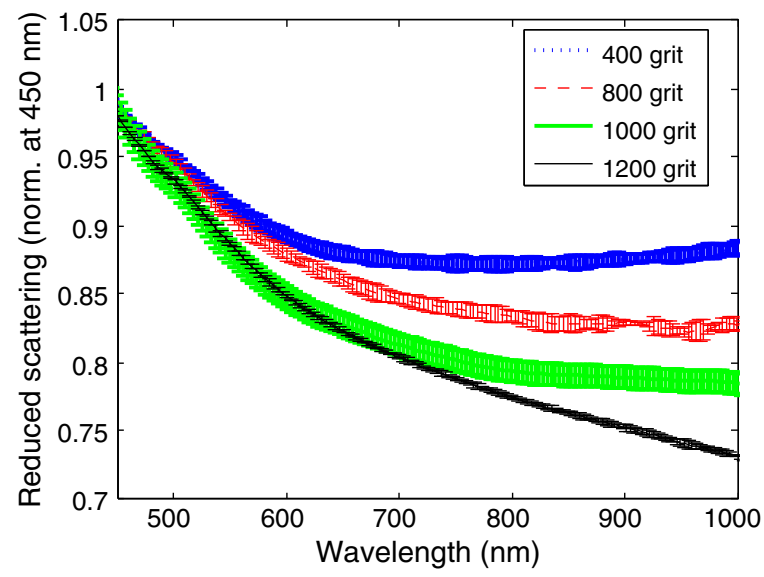

Fig. 3 Reduced scattering coefficient spectra as a function of grit size. Here, the mean spectra (normalized at $450 \mathrm{~nm}$ ) over every grit-specific concentration and measurement are reported. Error bars represent the standard deviation across each contributing spectrum $(n=9)$.

Table 3 Calculated scatter slope $(b)$ values resulting from least squares fit of normalized scattering spectra to a power-law Eq. (1).

\begin{tabular}{lcccccc} 
& $\begin{array}{c}400 \\
\text { grit }\end{array}$ & $\begin{array}{c}800 \\
\text { grit }\end{array}$ & $\begin{array}{c}1000 \\
\text { grit }\end{array}$ & $\begin{array}{c}1200 \\
\text { grit }\end{array}$ & $\mathrm{TiO}_{2}^{2,3,8}$ & Tissue $^{10,20}$ \\
\hline $\begin{array}{l}\text { "Scatter } \\
\text { slope" }(b)\end{array}$ & 0.007 & 0.11 & 0.15 & 0.32 & $\sim 1.25$ & $\sim 1.2 \pm 0.23$ \\
\hline
\end{tabular}

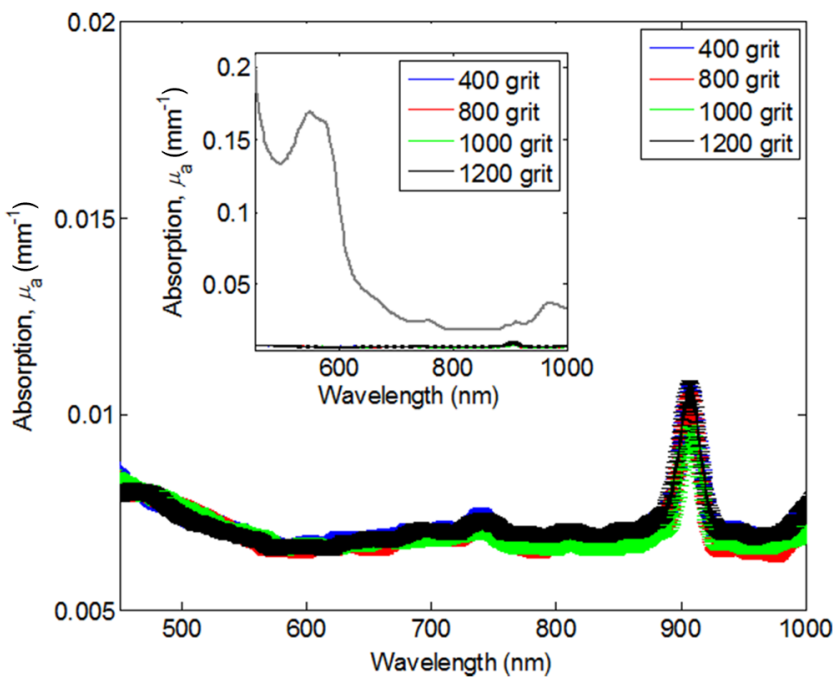

Fig. 4 Absorption spectra from $\mathrm{Al}_{2} \mathrm{O}_{3}$ phantoms across all grit-size particle distributions. Inset figure scales the phantom absorption in the context of the magnitude of absorption from in vivo tissue, $90 \mathrm{~min}$ post superficial partial thickness burn in a preclinical rat burn model, IUCUC \#1999-2064.

normalization. There is no significant dependence of absorption on concentration of particles or grit size. In the context of the range of tissue absorption properties anticipated in clinical and preclinical measurements, these absorption contributions are negligible (inset figure in Fig. 4). This indicates that $\mathrm{Al}_{2} \mathrm{O}_{3}$ affects only the scattering properties of phantoms and not absorption.

\section{Discussion}

From this initial investigation, we have demonstrated a simple method for producing low-cost PDMS phantoms with deterministic variable scattering properties. Informed by previous in vivo studies of an array of pathologic tissues, these $\mathrm{Al}_{2} \mathrm{O}_{3}$ scattering agents present the opportunity to mimic scattering spectra where there is a shift toward larger scattering objects. (These are in contrast to $\mathrm{TiO}_{2}$ particles, which produce scattering properties similar to those encountered in normal tissues.) These $\mathrm{Al}_{2} \mathrm{O}_{3}$ phantoms can be used in homogeneous, semi-infinite sample studies or in relatively thin, layered constructs, to evaluate the accuracy of optical spectroscopic methods and the performance of instrumentation, under controlled experimental conditions. This has distinct advantages over device and/or optical model development via in vivo tissue (pre- or clinical study) as it minimizes sources of unrelated tissue variation from the complexity and biodiversity present in in vivo samples. As these phantoms are stable over time and independently verifiable, they can provide a surrogate gold standard for the determination of accuracy and detectability limits of detection sensitivity of instrumentation/technique prior to implementing devices to study samples having uncontrolled or poorly defined sources of biological variance.

There are limitations, however, to the performance of the particles in the context of large (micron-sized) objects found in tissue. It is clear from the microscopy images that these powders are highly irregular and complex. Therefore their scattering properties are not well described by Mie theory alone; rather, each particle represents a superposition of multiple Raleighand Mie-like scatterers. The experimentally measured scattering spectra, calculated through IAD, also support this qualitative assessment. The shape of the scattering spectrum suggests a dependence that could be better described by two power-law components where the Raleigh-like scattering component is evident in the 450 to $550 \mathrm{~nm}$ region; however, 550 to $1000 \mathrm{~nm}$ fits more to a Mie-like power law. The study that we have presented here is not focused on developing a model to fully characterize the multiple scattering cross-sections derived from the complex geometric structures of these particles. Our goal here has been to evaluate these particles in the context of diffuse scattering regimes. In order to advance our understanding of these phantoms within the context of subdiffusive light scattering interaction, ${ }^{21}$ a greater understanding and characterization of the wavelength-dependent variance of the index of refraction of these particles is needed. Additionally, the implications of how both particle geometry and index within a PDMS medium would affect scattering phase function will need to be characterized. Future work will be directed toward resolving those aspects. As an alternative to PDMS methods presented here, methods using epoxy resin-based phantoms that utilize both $\mathrm{TiO}_{2}$ and $\mathrm{Al}_{2} \mathrm{O}_{3}$ to adjust subdiffusive scattering properties have been recently published. ${ }^{22}$

There are commercially available particles with distinct micron-sized distribution that do not suffer from the complex geometric shapes exhibited by these $\mathrm{Al}_{2} \mathrm{O}_{3}$ particles. $^{23}$ Whereas these scattering materials, which include monodisperse latex and polystyrene microspheres, represent scattering particles that are more easily matched to Mie theory, there were multiple reasons that we have not chosen to use them in PDMS phantoms. Unlike $\mathrm{Al}_{2} \mathrm{O}_{3}$, the costs of these particles are several orders of magnitude greater, and for large volumes that would be necessary within the context of wide-field 
imaging, which is our primary interest, these would be cost prohibitive to use. For example, the total phantom cost, in the typical volumes considered in this study, is estimated to be greater than $\$ 1000$ for materials alone (versus $>5$ when $\mathrm{Al}_{2} \mathrm{O}_{3}$ is used). Furthermore, the index of refraction of these microspheres tends to be very close to that of PDMS and therefore presents a challenge to mimic the magnitude and scatter anisotropy of tissue and/or oxide-based scattering agents. To that end, these particles are typically submerged in water (lower index than tissue/ PDMS); however, that presents yet another challenge to integrating them in PDMS as it is hydrophobic.

Whereas $\mathrm{Al}_{2} \mathrm{O}_{3}$ particles may not be a universal surrogate for the scattering changes in tissue and tissue pathologies, they do exhibit a clear and deterministic power-law dependence correlated with mean particle size distribution within a wavelength range of 550 to $1000 \mathrm{~nm}$. These scattering phantoms present an opportunity to study specific tissue pathologies, where there are increases in scatter object size and depth-specific changes within total interrogation volume. ${ }^{8}$ As with their $\mathrm{TiO}_{2}$ scattering agent counterparts, $\mathrm{Al}_{2} \mathrm{O}_{3}$ particles are relatively inert and nonreactive within PDMS, which allows these phantoms to be stable over time.

\section{Conclusion}

This investigation has demonstrated that $\mathrm{Al}_{2} \mathrm{O}_{3}$ powders can be used to approximate a range of micron-sized scattering particle distributions within a PDMS matrix. This new class of scattering agents can provide stable, physical constructs that simulate wavelength-dependent changes in light transport and scattering correlated to structural changes in tissue due to a variety of tissue pathologies, such as burn wounds, cancerous tumors, or lesions. Based on independent measurements of the optical properties of these phantoms, each grit-size of $\mathrm{Al}_{2} \mathrm{O}_{3}$ exhibited a unique power-law spectral dependence that agrees with the expected scattering from the relative differences in particle size.

\section{Acknowledgments}

The authors gratefully acknowledge funding provided by NIH/NIBIB Grant No. R01GM108634-01A, the NIH NIBIB Biomedical Technology Research Center (LAMMP: P41EB015890), the Military Photomedicine Program, AFOSR Grant No. FA9550-10-1-0538, and the Arnold and Mabel Beckman Foundation.

\section{References}

1. B. W. Pogue and M. S. Patterson, "Review of tissue simulating phantoms for optical spectroscopy, imaging and dosimetry," J. Biomed. Opt. 11(4), 041102 (2006).

2. A. M. Grant et al., "Diffuse optical spectroscopy of melanoma-simulating silicone phantoms," Proc. SPIE 7187, 718702 (2009).

3. F. Ayers et al., "Fabrication and characterization of silicone-based tissue phantoms with tunable optical properties in the visible and near infrared domain," Proc. SPIE 6870, 687007 (2008).
4. F. P. Bolin et al., "Refractive-index of some mammalian-tissues using a fiber optic cladding method," Appl. Opt. 28(12), 2297-2303 (1989).

5. R. Bays et al., "Three-dimensional optical phantom and its application in photodynamic therapy," Lasers Surg. Med. 21(3), 227-234 (1997).

6. D. K. Cai et al., "Optical absorption in transparent PDMS materials applied for multimode waveguides fabrication," Opt. Mater. 30(7), 1157-1161 (2008).

7. H. Jang, T. J. Pfefer, and Y. Chen, "Solid hemoglobin-polymer phantoms for evaluation of biophotonic systems," Opt. Lett. 40(18), 43214324 (2015).

8. R. B. Saager et al., "Multi-layer silicone phantoms for the evaluation of quantitative optical techniques in skin imaging," Proc. SPIE 7567, 756706 (2010).

9. M. Firbank, M. Oda, and D. T. Delpy, "An improved design for a stable and reproducible phantom material for use in near-infrared spectroscopy and imaging," Phys. Med. Biol. 40(5), 955-961 (1995).

10. R. B. Saager et al., "Quantitative near infrared spectroscopic analysis of Q-Switched Nd:YAG treatment of generalized argyria," Lasers Surg. Med. 45(1), 15-21 (2013).

11. J. R. Mourant et al., "Mechanisms of light scattering from biological cells relevant to noninvasive optical-tissue diagnostics," Appl. Opt. 37(16), 3586-3593 (1998).

12. J. Q. Nguyen et al., "Spatial frequency domain imaging of burn wounds in a preclinical model of graded burn severity," J. Biomed. Opt. 18(6), 066010 (2013).

13. I. Georgakoudi et al., "Fluorescence, reflectance, and light-scattering spectroscopy for evaluating dysplasia in patients with Barrett's esophagus," Gastroenterology 120(7), 1620-1629 (2001).

14. V. Krishnaswamy, "Quantitative imaging of scattering changes associated with epithelial proliferation, necrosis, and fibrosis in tumors using microsampling reflectance spectroscopy," J. Biomed. Opt. 14(1), 014004 (2009).

15. A. Cerussi, "In vivo absorption, scattering, and physiologic properties of 58 malignant breast tumors determined by broadband diffuse optical spectroscopy," J. Biomed. Opt. 11(4), 044005 (2006).

16. A. Ponticorvo et al., "Quantitative assessment of graded burn wounds in a porcine model using spatial frequency domain imaging (SFDI) and laser speckle imaging (LSI)," Biomed. Opt. Express 5(10), 34673481 (2014).

17. J. Q. Nguyen et al., "Quantitative longitudinal measurement in a rat model of controlled burn severity using spatial frequency domain imaging," Proc. SPIE 8565, 85650S (2013).

18. A. J. Moy et al., "Optical properties of mouse brain tissue after optical clearing with FocusClear (TM)," J. Biomed. Opt. 20(9), 095010 (2015).

19. S. A. Prahl, M. J. C. Vangemert, and A. J. Welch, "Determining the optical-properties of turbid media by using the adding-doubling method," Appl. Opt. 32(4), 559-568 (1993).

20. S. H. Tseng, A. Grant, and A. J. Durkin, "In vivo determination of skin near-infrared optical properties using diffuse optical spectroscopy," J. Biomed. Opt. 13(1), 014016 (2008).

21. S. C. Kanick et al., "Sub-diffusive scattering parameter maps recovered using wide-field high-frequency structured light imaging," Biomed. Opt. Express 5(10), 3376-3390 (2014).

22. P. Krauter et al., "Optical phantoms with adjustable subdiffusive scattering parameters," J. Biomed. Opt. 20(10), 105008 (2015).

23. I. Polysciences, Polybead Microspheres, 2011, http://www.polysciences. com/skin/frontend/default/polysciences/pdf/Polybead_Microspheres.pdf (15 March 2016).

Biographies for the authors are not available. 